\title{
Individual Predisposition for Learning and Neuroplasticity
}

\author{
Sibylle C. Herholz \\ German Center for Neurodegenerative Diseases (DZNE), 53175 Bonn, Germany \\ Review of Ventura-Campos et al.
}

In a popular novel for young adults, the first book of the Harry Potter series, students starting their school career undergo a ritual: They put on a magical sorting hat that looks inside their minds for a few moments, determines the students' individual predispositions and latent abilities, and assigns them to a "house" of the school that will best develop those tendencies (Rowling, 1997). Although such a method for discerning people's abilities does not exist in real life, scientific interest in the neural networks that determine individual predisposition for learning has recently surged, along with an increased awareness in cognitive neuroscience that individual brain characteristics underlie the huge variability in behaviors and abilities (Kanai and Rees, 2011). Several studies have reported functional and structural brain characteristics that partly determine our learning potential in the auditory domain. For example, responsiveness of auditory cortex (AC) to finegrained pitch differences predicts learning rates in a subsequent melodic discrimination task (Zatorre et al., 2012a), and morphology and volume of Heschl's gyrus may predict the ability to learn speech

Received July 29, 2013; revised Aug. 22, 2013; accepted Aug. 23, 2013. I thank Karl Herholz and Robert Zatorre for helpful comments on a previous draft of this paper.

Correspondence should be addressed to Sibylle C. Herholz, Deutsches Zentrum für Neurodegenerative Erkrankungen e.V., Holbeinstrasse 13-15, 53175 Bonn, Germany. E-mail: sibylle.herholz@dzne.de.

DOI:10.1523/JNEUROSCI.3197-13.2013

Copyright $\odot 2013$ the authors $\quad 0270-6474 / 13 / 3315321-03 \$ 15.00 / 0$ contours and contrasts of a foreign language (Golestani et al., 2007; Wong et al., 2008). These recent findings about predisposition complement the more extensive literature on brain plasticity associated with learning (for a recent review, see Zatorre et al., 2012b). While studies comparing experts and novices cannot provide insight into preexisting differences because participants come pretrained, longitudinal experimentally controlled training studies can distinguish training-induced change from predisposition.

In a recent paper, Ventura-Campos et al. (2013) conducted such a longitudinal study and reported that correlated activity between speech-related brain areas during rest predicted subsequent auditory learning. In two functional magnetic resonance imaging (fMRI) experiments, native Spanish speakers were trained on phonetic discrimination. They learned the Hindi dental-retroflex place-of-articulation contrast: a challenging task because this contrast does not exist in the Spanish language. Participants learned to discriminate the sounds in a computer-based training that did not require them to produce the new speech sounds themselves. Training in the first experiment involved six 1 h sessions spread over 2 weeks. Resting state fMRI (brain activity at rest) and task-related fMRI (brain activity during the same phonetic discrimination task as in training) were measured before and at the end of the training period. The second experiment was only intended to confirm the predictive finding of the first, and thus included a resting state measurement only before a shorter, massed training of 75 min on a single day.

The main finding of the paper was that resting-state correlations of activity between left anterior insula/frontal operculum and left superior parietal lobe predicted learning success on the phonetic training as measured by posttraining discrimination performance. Both of these regions are part of the language network that is involved in processing of familiar and new speech sounds and that was identified in the basic phoneme listening contrast. Intriguingly, although correlations decreased with learning, especially in participants who learned well, those with the highest correlations to begin with were the best learners. There was a clear relationship across the whole group, but it should be noted that it would not be strong enough to allow prediction at an individual level. A feature of Ventura-Campos et al.'s (2013) paper that merits special recognition is the immediate replication of the finding in the second experiment, with a new sample and with a slightly different training protocol, speaking for the reliability of the predictive relationship.

These new results extend our knowledge about predisposition for auditory learning, and importantly go beyond the level of cortical regions to the level of cortical networks. However, given the previous results about predictive value of AC morphology and function (Golestani et al., 2007; Wong et al., 2008; Zatorre et al., 2012a) along with Insula and 
parietal lobe white matter characteristics (Golestani et al., 2007), it seems surprising at first that the predictive functional network identified by Ventura-Campos et al. (2013) did not include the AC. This difference can be attributed partly to the methodological approach: The resting-state correlations that were used for prediction of learning were computed only between selected regions of interest (ROI). This approach allows more sensitive and targeted analysis within a known task-relevant network, but at the risk of omitting important network nodes that do not show up in specific task-related contrasts. In this case, ROIs were chosen based on the results of the initial taskrelated fMRI data analysis, specifically related to training-related change during Hindi phoneme perception and to processing a native phoneme contrast. AC did not show up in these specific contrasts although it was part of the basic activity pattern related to Hindi phoneme perception, and was thus not part of the uncovered functional network.

Would AC be expected to be part of the network? Previous results in humans (Golestani et al., 2007; Wong et al., 2008; Zatorre et al., 2012a), animal studies and anatomical analyses of AC connectivity (Scheich et al., 2011) indicate an important role of AC in various auditory learning tasks. Models both of speech processing and learning (Rauschecker and Scott, 2009) and of auditory learning (Scheich et al., 2011) include feedforward and feedback loops between auditory and other cortical structures. Based on these theoretical models and empirical findings, one might predict that the strength of connections between $\mathrm{AC}$ and other higher-order speech-relevant cortical areas should indeed be predictive of phonetic learning, with the assumption that the quality of the signal that is transmitted from auditory cortex to later processing stages is important for learning. In fact, a very recent study demonstrated that auditory learning of new words in an artificial language is predicted independently both by functional correlation and by integrity of structural connections between auditory and inferior frontal brain areas via the long segment of left arcuate fasciculus (Lopez-Barroso et al., 2013). Thus, the combination of functional and structural analyses can yield complementary results that expand our understanding of the individual variations in the networks that predetermine auditory learning abilities. Regarding the correspondence of func- tional correlation and structural connections, Ventura-Campos et al. (2013) also suggest that correlation strengths of anterior insula/frontal operculum with superior parietal cortex on a functional level may be related to underlying anatomical white matter connections demonstrated in anatomical studies (Martino et al., 2010).

The relative importance of inferior prefrontal and parietal areas together with AC might also depend on specific task characteristics. The task used by VenturaCampos et al. (2013) involved purely auditory perceptual learning. However, in natural environments we typically learn about new sounds in association with sensory input from other modalities, or by creating them ourselves (Scheich et al., 2011). Different cross-cortical connections may have relatively more important roles in learning by shaping through passive exposure versus feedback/error-based active learning, and depending on whether feedback is processed during or after training. In Ventura-Campos et al.'s (2013) study, participants received feedback about their auditory discrimination performance during training after each trial, but did not learn to articulate the sounds. Thus, articulation training of non-native phonemes or a comparison of auditory versus articulation training could be an interesting follow up study. Again, based on task characteristics and known anatomical connections, relatively concrete hypotheses can be made: strength of auditoryprefrontal-parietal connections should be of higher predictive value for auditory learning (Scheich et al., 2011), whereas auditorymotor connections should be relatively more important for predicting learning success in active articulation training (Rauschecker and Scott, 2009).

A promising aspect of the work by Ventura-Campos et al. (2013) is the task-independent nature of the applied brain imaging method. The advantage of resting state $\mathrm{AMRI}$ is that no tasks have to be specified in advance, and that the data acquisition is rather simple. Interestingly, pretraining behavioral performance was not predictive of subsequent learning, indicating that neuroimaging markers might be more sensitive than behavioral indices. We are far from fully exploiting all the information contained in these resting-state data. Still, to better understand resting-state measures, they can and should also be complemented by data on task-related activity and on anatomy. Rather than distinguishing good learners from bad learners on a single training paradigm, future studies might use the same datasets to identify correlations associated with differential preparedness for different types of learning, or for individually selecting the best training approach among several available in an educational or clinical setting. Here, first steps toward individualized approaches are already being made. For example, structural integrity of white matter fiber tracts predicted success in a cognitive rehabilitation intervention (Wolf et al., 2012), and morphology of auditory cortex to some extent relates to the career choice to become a phonetician (Golestani et al., 2011).

Finally, predisposition should not be confused with determinism. Even if our brains are not optimally wired to learn a certain task, we may still invest our motivation and energy into activities of our choice and compensate by increased effort and training intensity. The role of reward networks for predicting learning outcomes in different training approaches is yet unknown. Also, the fact that the correlated activity between anterior insula and parietal cortex in Ventura-Campos et al.'s (2013) study not only predicted learning success but also subsequently changed through training suggests that we may in fact modify our learning potential for future tasks by previous learning experiences. We're far from donning a magic hat to determine our individual potential, but the work by Ventura-Campos et al. (2013) takes us a big step forward to a differentiated view of predisposition for learning and brain plasticity.

\section{References}

Golestani N, Molko N, Dehaene S, LeBihan D, Pallier C (2007) Brain structure predicts the learning of foreign speech sounds. Cereb Cortex 17:575-582. CrossRef Medline

Golestani N, Price CJ, Scott SK (2011) Born with an ear for dialects? Structural plasticity in the expert phonetician brain. J Neurosci 31:4213 4220. CrossRef Medline

Kanai R, Rees G (2011) The structural basis of inter-individual differences in human behaviour and cognition. Nat Rev Neurosci 12:231242. CrossRef Medline

López-Barroso D, Catani M, Ripollés P, Dell'acqua F, Rodríguez-Fornells A, de DiegoBalaguer R (2013) Word learning is mediated by the left arcuate fasciculus. Proc Nat Acad Sci U S A 110:13168-13173. CrossRef Medline

Martino J, Brogna C, Robles SG, Vergani F, Duffau $H$ (2010) Anatomic dissection of the inferior fronto-occipital fasciculus revisited in the lights of brain stimulation data. Cortex 46: 691-699. CrossRef Medline

Rauschecker JP, Scott SK (2009) Maps and streams in the auditory cortex: nonhuman pri- 
mates illuminate human speech processing. Nat Neurosci 12:718-724. CrossRef Medline

Rowling JK (1997) Harry Potter and the Philosopher's Stone. London, UK: Bloomsbury.

Scheich H, Brechmann A, Brosch M, Budinger E, Ohl FW, Selezneva E, Stark H, Tischmeyer W, Wetzel W (2011) Behavioral semantics of learning and crossmodal processing in auditory cortex: the semantic processor concept. Hear Res 271:3-15. CrossRef Medline

Ventura-Campos N, Sanjuán A, González J, Palomar-García MÁ, Rodríguez-Pujadas A, Sebastián-Gallés N, Deco G, Ávila C (2013)
Spontaneous brain activity predicts learning ability of foreign sounds. J Neurosci 33:92959305. CrossRef Medline

Wolf D, Fischer FU, Fesenbeckh J, Yakushev I, Lelieveld IM, Scheurich A, Schermuly I, Zschutschke L, Fellgiebel A (2012) Structural integrity of the corpus callosum predicts long-term transfer of fluid intelligence-related training gains in normal aging. Hum Brain Mapp. Advance online publication. Retrieved September 11, 2012. doi:10.1002/hbm. 22177. CrossRef Medline

Wong PC, Warrier CM, Penhune VB, Roy AK,
Sadehh A, Parrish TB, Zatorre RJ (2008) Volume of left Heschl's gyrus and linguistic pitch learning. Cereb Cortex 18:828-836. CrossRef Medline

Zatorre RJ, Delhommeau K, Zarate JM (2012a) Modulation of auditory cortex response to pitch variation following training with microtonal melodies. Front Psychol 3:544. CrossRef Medline

Zatorre RJ, Fields RD, Johansen-Berg H (2012b) Plasticity in gray and white: neuroimaging changes in brain structure during learning. Nat Neurosci 15:528-536. CrossRef Medline 